\title{
High-order harmonic generation traces ultrafast coherent phonon dynamics in $\mathrm{ZnO}$
}

\author{
Richard Hollinger ${ }^{1,2}$, Valentina Shumakova ${ }^{3}$, Audrius Pugžlys ${ }^{3}$, Andrius Baltuška ${ }^{3}$, Sherzod \\ Khujanov $^{1}$, Christian Spielmann ${ }^{1,2}$, and Daniil Kartashov ${ }^{1, *}$ \\ ${ }^{1}$ Institute of Optics and Quantum Electronics, Abbe Center of Photonics, Friedrich-Schiller- \\ University Jena, 07743 Jena, Germany \\ ${ }^{2}$ Helmholtz-Institut Jena, 07743 Jena, Germany \\ ${ }^{3}$ Photonics Institute, TU Wien, 1040 Vienna, Austria
}

\begin{abstract}
Ultrafast coherent phonon dynamics in $\mathrm{ZnO}$ is studied via high-order harmonic generation by intense mid-IR laser pulses. We show, the phonon dynamic is very different after excitation in the tunnel and multiphoton regime.
\end{abstract}

High-order harmonic generation (HHG) in solids is a rapidly growing field of research in modern ultrafast nonlinear optics with potential for realizing solid-state attosecond sources $[1,2]$, reconstruction of the electronic band structure in semiconductors with alloptical methods $[3,4]$ etc. Numerous experiments, investigating the dependence of HHG on the intensity and polarization of the fundamental field, as well as polarization properties of the generated harmonics, were reported very recently. The capability of using HHG for time-resolved studies of ultrafast carrier dynamics in solids has been experimentally demonstrated using strong-field $\mathrm{THz}$ sources [5-7]. Here we report on the very first experimental results for using optical HHG to trace ultrafast phonon dynamics in bulk solids.

The pump-probe experiments are carried out with $100 \mathrm{fs}$ duration, $60 \mu \mathrm{J}$ energy laser pulses at $3.9 \mu \mathrm{m}$ central wavelength generated by a KTA-crystal based optical parametric amplification (OPA) system pumped by a high energy $\mathrm{Yb}: \mathrm{CaF}_{2}$ laser. The output of the OPA was split in a pump and probe arm by a 45:55 beamsplitter as the energy and the polarization of the pump and the probe beams was adjusted independently. The pump and probe beams are separately focused under an angel of $10^{\circ}$ using $\mathrm{f}=10 \mathrm{~cm} \mathrm{CaF}$ lenses to a radius of $65 \mu \mathrm{m}$ on the surface of a c-plane oriented, $100 \mu \mathrm{m}$ thick single crystalline $\mathrm{ZnO}$ slab (Crystec $\mathrm{GmbH})$. The temporal delay between pump and probe pulses was set by a motorized delay line with a minimum step size of $3 \mathrm{~nm}$. The spectral intensities of harmonics up to $21^{\text {st }}$ order, generated in the bulk $\mathrm{ZnO}$ by the probe pulse, were measured as a function of the pump-probe delay, intensity and polarization of the pump beam using a UV-visible spectrometer (Ocean Optics, USB 4000). All experiments were carried out at room temperature and ambient atmosphere.

\footnotetext{
* Corresponding author: daniil.kartashov@uni-jena.de
} 
A typical high order harmonic spectrum generated by probe pulses with the intensity $1 \mathrm{TW} / \mathrm{cm}^{2}$ in the unperturbed $\mathrm{ZnO}$ crystal is shown in Fig.1a. A strong peak near the $9^{\text {th }}$ harmonic is the photoluminescence emission at the $390 \mathrm{~nm}$ due to electron-hole recombination. The distinct maximum in the photoluminescence emission at zero delay is used for delay calibration. Figure $1 \mathrm{~b}$ ) shows the spectrally integrated signal of the $5^{\text {th }}$ and $21^{\text {st }}$ harmonics as a function of the pump-probe delay for a moderate pump intensity $0.57 \mathrm{TW} / \mathrm{cm}^{2}$ and parallel polarizations in the pump and probe beams. Here negative delays correspond to probe pulse arriving before the pump, thus showing harmonic yield in the unexcited bulk $\mathrm{ZnO}$. When both pulses are temporally overlapped, a strong damping of the yield is detected in all harmonics except for the $3^{\text {rd }}$ which shows an increased yield. When the probe pulse arrives after the pump and harmonics are generated in the excited semiconductor, oscillations are clearly visible in the measured harmonics yield (Fig.1a). Note, these oscillations are observed for all measured harmonics including the $3^{\text {rd }}$ harmonic. Fourier analysis of the data reveal an oscillation frequency of $2.8 \pm 0.2 \mathrm{THz}$ corresponding to the transversal optical $\mathrm{E}_{2}{ }^{\text {low }}$ phonon mode in $\mathrm{ZnO}$ (Fig.1c) [8]. The oscillations are clearly damped with the damping rate $\approx 1.6 \mathrm{ps}$ which agrees well with the electron-phonon scattering rate in $\mathrm{ZnO}$ [9]. As shown in Fig 1d,e the modulation amplitude of the harmonic yield has its maximum if pump-and probe beam polarization are parallel, and drops abruptly with increasing the angle between the two polarization. Varying the pump pulse intensity from $0.2 \mathrm{TW} / \mathrm{cm}^{2}$ to $1 \mathrm{TW} / \mathrm{cm}^{2}$ we observe a softening of the phonon by lowering the frequency from 2.95 to $2.8 \mathrm{THz}$. At the same time the oscillation amplitude is increased by a factor of eight.

We repeated the pump-probe experiment using 80 fs laser pulses at $0.8 \mu \mathrm{m}$ wavelength. Here, the third harmonic at $266 \mathrm{~nm}$ was the highest observed harmonic. Surprisingly, no oscillations in the yield of the third harmonic as a function of the delay were observed, although both the $3^{\text {rd }}$ and $15^{\text {th }}$ harmonics of the $3.9 \mu \mathrm{m}$ source (the last one is very close to $266 \mathrm{~nm}$ of the $3^{\text {rd }}$ harmonic of Ti:Sa) show clear modulation.

Our experimental observations can be explained as follows. The intense mid-IR pump pulse excites a coherent phonon wavepacket which dynamically modulates the electronic band structure, causing modulation in the intensity of the harmonic emission. It is
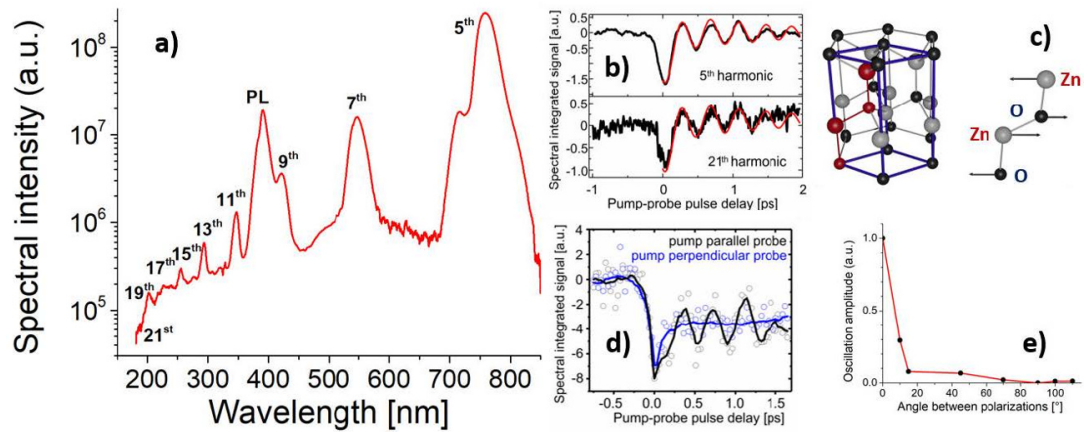

Fig. 1. a) Spectrum of high-order harmonics generated by the probe pulse in unperturbed $\mathrm{ZnO}$ crystal; b) spectrally integrated yield of the $5^{\text {th }}$ and $21^{\text {st }}$ harmonics as a function of the delay relative to the pump pulse. The pump and probe have the same polarization; c) Wurtzit crystal structure of $\mathrm{ZnO}$ with marked unit cell (blue), atomic basis (red) and the $\mathrm{E}_{2}{ }^{\text {low }}$ phonon mode; d) spectrally integrated yield of the $5^{\text {th }}$ and $21^{\text {th }}$ harmonics as a function of the delay for pump and probe beam with the same (black curve) and orthogonal polarizations (blue curve), respectively; e) Maximum amplitude of the oscillations of the $5^{\text {th }}$ harmonic yield as a function of the angle between the polarization of the pump and probe beams. 
commonly accepted that in transparent solids the mechanism of coherent phonon excitation by an off-resonant (photon energy is less than the bandgap) laser pulse is impulsive stimulated Raman scattering (ISRS) [10]. From the four possible Raman modes of optical phonons in $\mathrm{ZnO}$ [11], we see only a modulation of the harmonic yield at the frequency of the slowest $\mathrm{E}_{2}{ }^{\text {low }}(3 \mathrm{THz}$, oscillation period $\approx 334 \mathrm{fs}$ ) mode. This supports the assumption of ISRS as the excitation mechanism, because the $100 \mathrm{fs}$ duration of the pump pulse is larger than the vibrational period of the higher frequency modes (from 76 down to $58 \mathrm{fs}$ ). At the same time, the pump pulse excites electrons from the valence into the conduction band. Using the theory of strong field excitation in solids developed by Keldysh [12], we conclude that for the pump pulses at $3.9 \mu \mathrm{m}$ in an intensity range of $0.5-1 \mathrm{TW} / \mathrm{cm}^{2}$ the excitation proceeds in tunneling regime, whereas for the $0.8 \mu \mathrm{m}$ pulses in the same intensity range the excitation is dominated by three-photon absorption. For tunnel excitation, the photoelectrons in the conduction band remain cold by the end of the laser pulse [13], whereas three-photon absorption of $1.55 \mathrm{eV}$ quanta $(0.8 \mu \mathrm{m}$ wavelength) in $\mathrm{ZnO}$ (bandgap $3.37 \mathrm{eV}$ ) will result in the residual thermal energy of the excited electrons $\approx 1.3 \mathrm{eV}$. Therefore, in the latter case the phonon wavepacket will decay due to electron-phonon scattering on the time scale $\sim 100$ fs [14] shorter than the period of the $E_{2}^{\text {low }}$ mode. This might explain lack of modulation in the $3^{\text {rd }}$ harmonic yield in the experiments with pulses at $0.8 \mu \mathrm{m}$, but further numerical simulations are necessary for confirmation of this scenario.

This work is financially supported by the ACP Explore Project FSU-I-06-14.

\section{References}

1. S. Ghimire, G. Ndabashimiye, A. D DiChiara, E. Sistrunk, M. I Stockman, P. Agostini, L. F DiMauro, D. A Reis, J. Phys. B 47, 204030 (2014)

2. T. Higuchi, M. I. Stockman, P. Hommelhoff, PRL 113, 213901 (2014)

3. G. Vampa, T. J. Hammond, N. Thirè, B. E. Schmidt, F.Lègarè, C. R. McDonald, T. Brabec, D. D. Klug and P. B. Corkum, PRL 115, 193603 (2015)

4. A. A. Lanin, E. A. Stepanov, A.B. Fedotov and A. M. Zheltikov, Optica 4, 516 (2017)

5. B. Zaks, R. B. Liu, M. S. Sherwin, Nature 483, 580 (2012)

6. M. Hohenleutner, F. Langer, O. Schubert, M. Knorr, U. Huttner, S. W. Koch, M. Kira, R. Huber, Nature 523, 572 (2015)

7. F. Langer, M. Hohenleutner, C. P. Schmid, C. Poellmann, P. Nagler, T. Korn, C. Schüller, M. S. Sherwin, U. Huttner, J. T. Steiner, S. W. Koch, M. Kira, R. Huber, Nature 533, 225 (2016)

8. I. H. Lee, K. J. Yee, K. G. Lee, E. Oh, D. S. Kim and Y. S. Lim, Journ. Appl. Phys. 93, 4939 (2003)

9. J. Takeda, H. Jinnouchi, S. Kurita, Y. F. Chen and T. Yao, Phys. Stat. Sol. B 229, 877 (2002)

10. S. De Silvestri, G.Cerullo and G. Lanzani, Coherent Vibrational Dynamics, (Taylor\&Francis Group, New York, 2008)

11. T. C. Damen, S. P. S. Porto, B. Tell, Phys. Rev. 142, 570 (1966)

12. L. V. Keldysh, Sov. Phys. JETP 20, 1307 (1965)

13. V. D. Mur, S. V. Popruzhenko, V. S. Popov, JETP 92, 777 (2001)

14. C. Chen, M. Dutta, M. A. Stroscio, Phys. Rev. B 70, 075316 (2004) 